\title{
Knowledge Activation for Patient Centered Care: Bridging the Health Information Technology Divide
}

\author{
Sajda Qureshi \\ University of Nebraska Omaha \\ squreshi@unomaha.edu
}

\author{
Cherie Noteboom \\ Dakota State University \\ Cherie.Noteboom@dsu.edu
}

\begin{abstract}
The provision of healthcare is a collaborative process. It follows evidence based treatments which are becoming increasingly data driven and focusing on the best clinical outcomes. Patient centered care requires participation of patients in the decision making of the best treatment options. Healthcare provision requires both evidence based and patient centered care. In practice, these two perspectives conflict with each other due to the use of an information technology designed primarily for billing purposes. Using the knowledge activation framework developed by Qureshi and Keen [25], we analyze data from two hospitals in the Midwest that aim to achieve quality of care outcomes mandated by the Affordable Care Act. Following a grounded theory analysis of the focus group sessions we discover knowledge activation processes that may help overcome the divide between patient and evidence based care.
\end{abstract}

\section{Introduction}

Even though healthcare provision is a very collaborative process, the rise of data analytics and multiple treatment options has meant that physicians are faced with two conflicting ways of carrying out the treatment process. The first approach focuses on offering physicians the best available evidence about the most effective treatment for their patients. Evidence-based treatment options, also known as the data-driven approach to healthcare, rely on a combination of data from tests and bio-medical databases on a condition and scientific evidence. Such medicine is disease-oriented evidence based on randomized clinical trials. Patient-centered medicine focuses on patient participation in clinical decision making by taking into account the patients' perspective, and tuning medical care to the patients' needs and preferences [6, 29].

While both approaches are valuable, they are in conflict with each other when EHRs are used as an integral part of the healthcare provision process. Both approaches require knowledge to be activated from different specialists and providers involved in treating a single patient. The knowledge activation perspective used in this paper by Qureshi and Keen [25] posits that knowledge identities are activated every time there is demand for it. This perspective focusses on 'knowledge in action' required to bridge the gap between patient centered care and evidence based care. The question investigated in this paper is: what are the knowledge activation processes that need to be supported by Heath Information Technology (HIT) to bridge this gap?

Patient centered care has increased in importance in recent years with the passing of the Patient Protection and Affordable Care Act of 2010, which mandated the integration of physician quality reporting and Electronic Health Record (EHR) reporting and increased patient centered care measured through "meaningful use" practices. This Act required the creation of measures and reporting of the "meaningful use of the electronic health record" and "quality of care furnished to an individual." In doing so, the law links the adoption of the electronic health record with the measurement of quality of care to the patient through objectives that measure the adoption of technology by eligible providers. Core objectives of the act include identifying basic functions that enable EHRs to support improved health care, progress toward supporting advanced processes such as providing patients with increased online access to their records and measuring improved outcomes [7].

The EHR technology is mandated by the Health Information Technology for Economic and Clinical Health Act (HITECH). This act authorizes incentive payments through Medicare and Medicaid to clinicians and hospitals when they use EHRs privately and securely to achieve specified improvements in care delivery. In addition, major incentives are available through the American Recovery and Reinvestment Act, passed in February 2009, which included a very large stimulus payment for eligible providers, hospitals and physicians for the adoption of EHRs.

Despite the above legislation, medical care continues to struggle with patient centered care as any feedback from the patients to the physicians is made more difficult 
because of the EHRs. As a consequence of the above legislation the provision of healthcare has become centered on the use of the EHRs which primarily support billing and insurance reimbursement requirements. As the EHRs are primarily designed to support evidence based care, they offer different healthcare professionals specific views that are unique to their specialties. None are able to see a complete view of the patient's record. A physician's orders are entered into a patient's chart on the EHR system. This triggers responses from relevant specialties to act on the patient. If a specialist is unable to complete a physician's order, or the patient is unable to tolerate a medication or treatment, the specialist will have to contact the physician outside the system to receive further instructions. Thus patient centered care takes place outside the EHRs.

Often patient centered care requires verbal communication and contact with the patient together with the physician and relevant specialties. Evidence based care is supported by the EHRs to the extent that treatments are entered into the system, physicians can use the data contained in it to make treatment decisions. Both forms of care are required to achieve quality of care and both forms of care require knowledge activation. Patient centered care requires a different type of knowledge to be activated than does evidence based care. The processes of trial and error that leads to the most appropriate therapy for a patient can take time, effort and prove to be costly. We discuss this further in the theoretical background offer a view into the knowledge activation processes affecting healthcare providers as they use EHR's to offer healthcare.

\section{Theoretical Background}

Traditional medical practice is built upon evidence based medicine. Evidence-based medicine is the conscientious, explicit, and judicious use of current best evidence in making decisions about the care of individual patients. This approach considers the practice of medicine as a cognitive-rational enterprise. In this approach the uniqueness of patients, their individual needs and preferences, and their emotional status are easily neglected as relevant factors in decision-making. The practice of evidence based medicine means integrating individual clinical expertise with the best available external clinical evidence from systematic research [6, 29].

In recent years, medical diagnosis and treatment options have become data-driven. Given that physicians no longer have time to read the latest research on a condition, they rely on evidence from pharmaceutical research and other biomedical databases to arrive at the best option for treatment. This has meant that the cost of care has risen for the diagnosis, treatment and evaluation of the patient's condition. According to Clifton [10], healthcare in America costs 2.5 trillion dollars a year and is expected to grow to 4.5 trillion in six years. The Institute of Medicine [16] reported that the U.S. healthcare system is "fundamentally broken" and called on the Federal government to make a major investment in information technology in order to make the changes, such as the "commitment to technology to manage the knowledge bases and process of care" [16;178]. According to the Agency for Healthcare Quality and Research, automation is, in principle, able to improve the quality and safety of care delivered by healthcare facilities by enabling collaboration among physicians, medical personnel and patients.

Patient-Centered care is seen to be a natural progression towards greater efficiency and effectiveness in healthcare provision. This form of care is one in which the patient actively participates in his or her care, delivery of care takes place from a patient's point of view, there is greater communication with the patient, and therapy is tailored to the needs of the patient [20,30,31]. The implementation of HIT, in particular the Patient Health Record (PHR), may appear to enable greater patient centered care through better access to patient data, shorter recovery through targeted care, lower cost through fewer tests and increased meaningful use practices [7, 9, 31].

Patient centered care entails collaboration, communication and joint decision making with patients whose preferences are taken into account when developing treatment options suited to the needs of the patients and have reported improved health outcomes [5, 9 , 23]. The practice of evidence based medicine is about using individual clinical expertise with the best available external clinical evidence from systematic research such as clinical trials [6, 29]. Medical diagnosis and treatment options have become data-driven, requiring that physicians have access to the latest research on a condition and data on the patient's condition.

Collaboration is purposeful joint action through the construction of relevant meanings that are shared among members. In order to enable patients to become more involved in their care, beyond coordinating the different components of their care, the healthcare delivery process needs to be more centered on the patient with collaborative processes to enable the construction of relevant meanings. Patient centered care implies a paradigm shift in the relationship between doctors and patients, but also requires the development of patientoriented research [30]. The literature on patient centered care suggests that it requires customization of processes and treatment options to patient needs and health conditions. But there is little agreement as to what those patient centered outcomes are and how they should be 
assessed. This is due to the complexity of the healthcare delivery which is in a tension between routine and variable processes [[24, 28, 30,31].

Studies on the outcomes of patient centered care have reported better recovery from their discomfort and concern, better emotional health, and fewer diagnostic tests and referrals [9, 13, 23, 27]. Patient centered care relies on physicians capturing the benefit from the technology to collaborate with other medical practitioners ensuring that care is improved.

In practice, this is a challenge when physicians resist technology, rely on other medical personnel to communicate with the patients and are accustomed to offering standardized, evidence based therapies instead of those targeted to the patient's needs. The literature indicates physicians resist the technology due to productivity issues, workflow challenges, lack of support and other issues related to the mismatch between the technology and healthcare delivery process $[1,3,4,12,19,21,24,26]$. A recent study by Noteboom and Hafner [22] found that "technological upset" takes place when physicians do not unlearn their past behaviors in order to use technology. They report perceptions of unbalancing skills, stress, and frustration occurring while working with an EHR system.

Kane and Labianca [17] add that "patient-level data are particularly valuable for the quality of care metrics, because individual patient characteristics play a significant role in determining care results (e.g., how faithfully the patient follows the doctor's recommendations). If patients fail to manage their chronic diseases adequately, escalating conditions can become extremely expensive to treat and can significantly compromise the patient's quality of life." (17:510). From a public health perspective, patient centered care requires 'a partnership among practitioners, patients, and their families (when appropriate) to ensure that decisions respect patient's wants, needs, and preferences and that patients have the education and support they need to make decisions and participate in their own care" ([16, 28:7].

This enables lower cost preventive medicine to become an option for patients. Robinson et.al. offer an economic view of the patient as the informed consumer who makes decisions based on cost and quality of care. They also identify care from a patient's perspective to include "respect, courtesy, competence, efficiency, patient involvement in decisions, time for care, availability/accessibility, information, exploring patient's needs, and communication” [28:602].

Studies have found that when providers use Electronic Health Records to manage and monitor patients, their health outcomes do improve as long as the use of technology is accompanied by management of care $[8,15]$.

However, current medical work practices revolve around the providers, standardized practices and evidence based treatment options [1, 2, 11].Kane and Labianca [17] offer a multi-level view of Information Systems (IS) avoidance in healthcare groups and suggest that a key to enabling better use of the technology among and between healthcare providers and patients is by enabling people central to the healthcare group to overcome the detrimental effects on quality and efficiency. The avoidance of information systems by healthcare providers, patients and physicians is beyond simple non-use and has to do with a number of factors at the individual, group and organizational levels [11, 17, 24].

These factors affect IS avoidance at the patient level, doctor level and group level. There is IS avoidance at the physician level, as they control the information available to patients, their therapies and which additional healthcare professionals get involved in the patient's healthcare outcome. Thus limiting the patient's involvement in their own therapy. This leads to increasing the physicianpatient divide.

\subsection{Knowledge Activation in Healthcare}

Knowledge activation is "the conversion of knowledge into action.” Activating knowledge is about finding people with relevant knowledge and using it effectively through their willingness to provide, access, and share it as and when needed.” [25:41]. There are three types of knowledge identities that a person holds: 1) accountable which is part of individuals' professional lives; 2) discretionary which is theirs to share voluntarily; 3 ) autonomous which forms from their private experience. These identities determine the willingness of people to collaborate, communicate and share. This is illustrated in figure 1 below:

Figure 1: Knowledge activation in healthcare

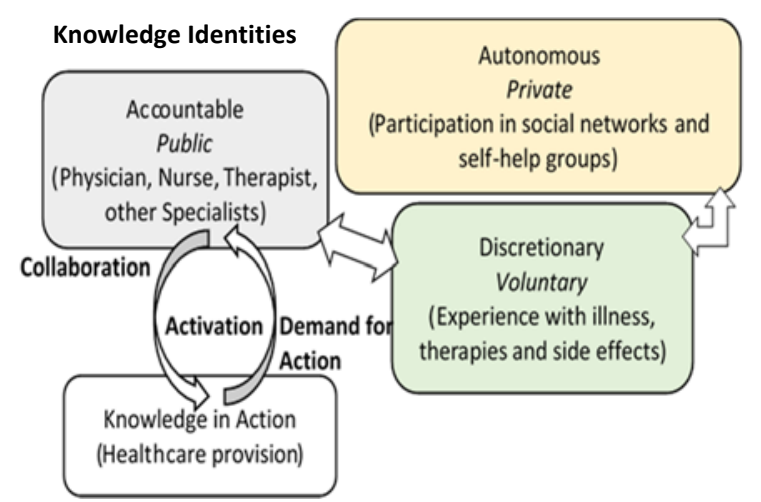

Source: Adapted from Qureshi and Keen [25]. 
Noteboom and Qureshi [21] found that successful adaptation of the EHR by physicians requires the capacity of physicians to activate all three levels of knowledge for use in their work processes. The physician's adaptation of the technology can enable better knowledge activation as they assess and verify the data, solve problems and find innovative solutions to the conditions for which there are few treatments.

Activating knowledge requires that there be demand for it. Patients create demand for a physician's knowledge by communicating their symptoms, interactions to therapies, side effects and other information needed by a physician to be able to arrive at a therapy. When a patient with symptoms that need treatment visits the physician, this creates demand for action. Demand for action triggers collaboration between people who then activate their knowledge to bring it into action.

Collaboration is purposeful joint action through the construction of relevant meanings that are shared among members. Collaboration is needed to: 1) determine what action is required and relevant; 2) identify knowledge to carry out a required action; 3) meet the demand for action [25]. There are many incentives to share accountable knowledge, which is part of responsibility and position. There is less incentive to share discretionary and autonomous knowledge, which is personal and in many instances can be tacit information the owner is unaware of possessing or the owner may carefully guard as a component of his or her identity.

Effective patient-centered care is about the identification of the best intervention for every individual patient using personalized medicine and tailored therapeutics [30]. This requires collaboration among healthcare providers, specialists and patients, including their representatives. In order for physicians and healthcare providers to provide care to patients, they need to bring their knowledge into action. At the same time, patients also have knowledge that needs to be activated in the provision of care. For example, in treating a patient with a known condition, a physician will activate their accountable knowledge and offer treatment for which evidence suggests is the best possible treatment for that condition. The patient's knowledge would also be activated to find out about any allergies and past medical history not documented in the system.

However, if the patient's discretionary knowledge on side effects or allergies to that treatment is not activated, then the patient's condition may worsen or even die. This is a result of evidence based treatment that did not receive patient input or input from a patient representative. This lack of activation leads to medical errors. Medical errors, according a recent Johns Hopkins study is the $3^{\text {rd }}$ cause of death in US hospitals [18]. Knowledge activation is a process that requires activation of appropriate knowledge identities between multiple individuals who collaborate with each other when bringing their knowledge into action. When the appropriate knowledge identities are activated, then the gap between patient centered and evidence based care is reduced, hence reducing medical errors.

\section{Methodology}

Evidence from two sets of data were used to study knowledge activation for patient centered care. The data for this study was gathered through a focus group conducted at a Federally Qualified Healthcare Center (FQHC) in the Midwest. This site was chosen for the study as they had achieved high scores for meaningful use using their EHR. The participants included providers from multiple specialties including psychologists, physicians, and community healthcare workers. The participants were asked about the challenges they faced as they used information technology to provide healthcare and what functionalities they would like to see in their EHRs.

In order to explain the categories, slices of data from the transcripts were identified. The transcripts from the focus group were triangulated with transcripts from a second study at an acute care county hospital located in the Midwestern United States. This hospital was chosen because of its central location and importance in providing healthcare for the county just like the FQHC use for the focus group. Data from two open ended questions that asked participants what challenges they faced with EHRs and what functionalities they would like to see. The participants in the study were identified as health care professionals or physicians, pharmacists, and residents, representing various different specialties in a Midwest medical center setting.

By using slices of data from transcripts of two studies, we are able to offer greater depth of insight into the categories and identify relationships between them. By expanding the scope of our analysis we can offer generalizability for the resulting theory. In the Grounded Theory Method, we extend theory through theoretical sampling [32].

The focus group data was then analysed using grounded theory open coding approach [14] to arrive at labels depicting the challenges and opportunities that healthcare professionals face as they offer care. Further grounded theory analysis lead to the discovery of categories of knowledge activation that may enable patient centered care and those that may hinder such care to take place. 


\section{Results}

We used the 68 open ended responses to two questions relating to the physicians' experiences with information technology in their provision of healthcare. In particular, their experiences related to their use of Electronic Health Records for the provision of evidence based care. Based on the knowledge activation framework described above, transcripts for each of the knowledge activation concepts as physicians use EHRs to provide care were selected. The physicians' responses are denoted with a "P". Their responses relate to the use of the EPIC system.

In addition to the responses from the survey, transcripts from the focus group session were used to identify knowledge activation issues and opportunities as specialists use EHRs to provide care. These responses from other specialties from the focus group are denoted with an "O". The responses of the other specialists relate to their use of the NextGen system. Both sets of transcripts are illustrated below under the concept that they represent.

\subsection{Activation of Accountable Knowledge}

In order to respond to demands for action for healthcare provision, accountable knowledge is the primary knowledge identity to be activated. Accountable knowledge is an integral part of a person's professional identity and is often formed through many years of education, training and is illustrated through certifications and degrees. Physicians and specialists draw upon on their accountable knowledge to be able to collaborate with each other in offering healthcare. The EHRs are supposed to assist in the provision of healthcare by offering a means through which the healthcare providers can share data to arrive better outcomes. The following transcripts illustrate the ways in which accountable knowledge is activated using the system:

P:"Having physician notes and labs readily available to see if patient is improving or not improving. Sometimes, therefore, these physician notes take a while to load. Seeing their home medications."

P: "Data widely available to health care givers responsible for patient care. Labs are available without having to hunt down a chart.”

$P$ : "Being able to quickly access information on a Pt's chart as it becomes available without having to be directly on the medical unit."
O:"..data you'll get our information, MRI, labs, and all that. We can make a connection, that's where the manual billing caps become very handy. In that you can use time. I spend about two to five minutes reviewing data and then justify what I did."

O: " Multiple charts easier to read on the computer, with EMR everything is there. Vitals, demographics, history. With little time, it's easier to do homework first, see patient, then return to do data entry."

The above transcripts suggest that the system is useful in the activation of accountable knowledge. At the same time, the responses indicate that physicians' activation of accountable knowledge for the provision of evidence based care was limited by the functionality of the electronic health record technology.

\subsection{Discretionary and Autonomous Knowledge}

Provision of patient-centered care involves the activation of discretionary and autonomous knowledge. Activation of discretionary knowledge is needed for a physician to be able to communicate with patients in order to understand their healthcare needs. Activation of autonomous knowledge involves the use of personal experiences and relationships to be able to work with patients and other providers in a manner that addresses patient needs. The transcripts below illustrate that the system imposes a structure on the healthcare provision process which restricts the activation of discretionary and autonomous knowledge identities of patients and the specialties involved in their care.

P:"Over documentation due to amount of documenting "radio" or click options."

$P$ : "Falsification of information - the templates given in the EHR system are unrealistic and require way too much time to actually ask all of the questions listed, therefore I think physicians will "assume" several of the questions on behalf of their patient, when in reality these questions have gone unasked."

$P$ : "Errors in order entry. "Downtime" when system not available with no alternative for information retrieval. Reduction of clinical information entry from informative text to non-informative and difficult to synthesize checkboxes."

O:" from the medical standpoint it's not really standardized. You have a complex that you can form but you still have no way to free text anything you want."

O:" using technology occasion when it's not user friendly, from the perspective of there's nothing natural about it. There's no real logical flow .. it looks like an IT person put it together." 
The above comments state that entering data in the system causes errors in the care of patients and takes away from valuable time needed to communicate with patients. This inhibits the activation of discretionary knowledge which takes place when patients and their providers are able to communicate with each other unencumbered by the technology. It is also clear from the above that there is no activation of autonomous knowledge through the system.

\subsection{Collaboration}

The process of creating relevant meanings that are shared among different members, involves collaboration. This involves purposeful joint action among the different healthcare providers. The following transcripts illustrate was in which the system enables collaboration among the different members of a healthcare team.

P: "Multiple providers can look at same chart at the same time. Order sets, if developed correctly, can be very efficient."

$P$ : "More than one personnel can look at a patient info at the same time."

$P$ : "I believe interfacing with pharmacies will be a huge benefit and will reduce med errors."

O:" Need to free text pertinent information but it doesn't get counted by the by the system. It almost forces you to go to the template and to answer those questions because otherwise you get dinged. I mean your efforts are not really seen."

O:" [Using Scribes].. is excellent. It works in the ER because it literally increases our productivity by anywhere from one half to one full patient per hour."

While they may be useful in sharing data, the EHRs restrict collaboration between patients and their care providers because they force data entry. Even after data has been entered it is not a guarantee that the data will get used as part of the treatment process. The data entry requirements for being part of a treatment process, require healthcare providers to work together outside the system.

\subsection{Demand for Action}

When using the system to provide care, the different healthcare providers need to be able to understand what is needed from them. Demand for action is about knowing what needs to be done and activating the appropriate knowledge identities. When asked how the healthcare providers would like to use the technology to be able to offer healthcare, they offered the following transcripts that illustrated demand for action:

P:“..immediate access to a patient's record by multiple members of the healthcare team; if pharmacy has a question regarding an order it is helpful that the nurse or physician can be looking at the same data where ever they might be - this can help speed up the process of clarifying questions related to med orders."

P: "Overall organization and ability to locate information quickly.”

O:" it would be nice if the system automatically link into the provider database to let us know if a patient is abusing medication. Or at least if there is a potential pattern."

O: "History, test results, information out there, research paper on their particular condition. So search for data but then data gets there on its own perhaps."

O: "Search function in a specific file. To be able to pull out anything where you could even tag sessions like you tag a picture."

The responses indicate that the availability of and access to labs and $x$-rays, drug interactions, flowsheet type reports and patient data from multiple locations was very valuable for the physicians. The use of technology to collaborate with other providers and respond to questions (demand for action) enables physicians to activate their accountable knowledge.

However, demand for action also requires the use of discretionary and accountable knowledge which need to be activated. Demand for action does not always lead to the activation of the appropriate knowledge identity, especially when that knowledge identity belongs to pharmacists who use a different part of the system. An outcome for demand for action is in prescription. The use of Electronic Health Records for prescriptions is very difficult as illustrated below:

P: “Approximately 20\% of drug orders require manipulation, time changes, allergy inquiry, order change, comments don't match the order, duplication removal, therapeutic inquiry by the pharmacist, and many other issues before the order is completed."

$P$ : "Often wrong information leads to incorrect meds"

$P$ : "The data is only as good as the people who enter it - for example heights and weights are frequently incorrect, this in turn messes up some fields that pull that data through into calculations of doses or creatinine clearance, etc. This could lead to potential med errors if the pharmacy staff would not happen to catch the error." 
The above transcripts illustrate yet another cause of medical errors due to unsupported activation by the system.

\subsection{Diagnosis}

One of the most important tasks in healthcare provision is diagnosis. The diagnosis and treatment processes involve activating accountable knowledge of the physician and the sharing of a patient's discretionary and autonomous knowledge in the diagnosis and treatment processes. The following transcripts illustrate the role of the system supporting diagnosis.

$P$ : "Any mistake, for instance diagnosis or other information, stays in the system forever."

$P$ : "The notes generated are difficult to read later - especially by someone unfamiliar with the format."

$P$ : "The record produced is often uninformative and unreadable (not illegible). The note produced by the EHR is wooden, has no flow and more closely resembles a ledger rather than a medical document. The only way to be able to document in a timely fashion is to prepopulate templates with information that may or may not be accurate. Most of the notes for a specific diagnosis produced by the EHR are similar if not identical to each other. "

$P$ : "System templates cause inaccurate notes. They are slowing ability to see patients and interfering with doctor patient relationship"

$P$ : "Efficiency, data entry is time consuming... documentation takes away from direct patient care.

P: "Time spent by providers attending the computer but not patients." care”

$P$ : "[The system takes]Time away from hands on

O:" also for behavioral health, there's times when HR systems want you to ask questions in a certain way and you can't go to the next question until you answer the first question. But sometimes the way that those questions are placed, it's wrong in terms of how you are asked."

O:" You have got to go to page six, put your diagnosis in the go back to page one because then you have all these reminders that come up"

Even though the electronic health records are intended to make it easier for physicians to accurately diagnose patients, the above comments suggest that in reality the opposite is the case. Over documentation, inaccuracies in the information entered and lack of inter-operability between the data in the system mean that physicians are unable to fully use their existing information systems to diagnose patients.

\subsection{Evaluation of Treatment options}

An important component of patient centered care is for physicians to be able to search and evaluate treatment options with the patient. The following transcripts offer insight into the extent to which the system enables this process.

P: "Searching for any particular lab in the part is not possible, unless you back in time. You see unwanted lab results also. Improving Searching to enable looking for one particular lab or medicine will be good. Can you install search for any particular event?"

$P$ : "Finding information is not always the easiest to get to. Does not seem to be consistent with how the drop down menu is organized. Alphabetical order would be great and also a menu/flow chart as to what data each section entails."

O:" using the EMR, we don't have a way to see all the information of the patient from another facility."

O: "Search function in a specific file. To be able to pull out anything where you could even tag sessions like you tag a picture. Like to be able to pull information."

As illustrated in the above transcripts, this is a collaborative process that is not well supported by the system.

\subsection{Implementation of Treatment}

Implementing treatments requires multiple knowledge identities of different healthcare providers. The following transcripts illustrate how the system is used to implement treatments:

$P:$ “...dictating physician plan of treatment and workup of patients with pre-set algorithms...[leads to] decreasing efficiency and interferes with timely patient care."

O: " another limitation is in the medication line..we discover that the patient's interaction with the medicine or whatever it is that we want to cancel that script. Although we did cancel it in our computer it doesn't cancel at the pharmacy.... you have to stop what you're doing, pick up the phone, wait for a pharmacist to get on the phone and then tell them know that you want to cancel that script."

O:" a clinical person does not want to spend five minutes looking for one test.. Because that's five minutes that we could have been discussing with a patient" 
O: "A lot of it is data driven because if you don't do your reminders then you're not doing what you're supposed to do! So according to whoever collected the data"

O:" Treatment needs to be completed for insurance and the questions are sensitive, with technology its hard to manage being there for the patient and use the technology at the same time."

As illustrated in the above transcripts the role of the system is primarily for billing purposes and justification of these options. According to the transcripts, the healthcare providers consider this to be a limitation in the provision of effective patient centered care.

\subsection{Assessment and Follow-up}

A different form of collaboration takes place at this stage. When healthcare providers need to followup with patients, information is needed from the system, other providers and patients to be able to assess outcomes. The use of EHRs for this process is illustrated below:

P:"I am less efficient as compared to dictating and writing orders. Unless you access to EHR, it is no more efficient than a paper chart (clinic to hospital and hospital to clinic integration).

$P$ :"Allows all users to quickly enter prespecified/template notes, which in turn results in in very little pertinent history and data in progress notes leading to little detail for visits."

O:" if a patient goes to a correction and was discharged, and was supposed to follow up say a week.. Comes to you and we don't have a way we can go to computer access their information from that. That is the biggest drawback in the computer right now."

While the system is supposed to track healthcare outcomes, the above transcripts illustrate

\section{Analysis}

In this section the analysis of the transcripts from the focus group sessions is reported. All the transcripts of the focus group session were labelled using grounded theory open coding. This processes helped us identify labels for categories of challenges identified above. A total of 100 labels were discovered. These were then grouped to the categories identified during the triangulation phase. These are illustrated as follows:

\begin{tabular}{|c|c|c|}
\hline Category & Sample Labels identified & $\mathbf{F}(\mathbf{x})$ \\
\hline $\begin{array}{l}\text { Activation of } \\
\text { Accountable } \\
\text { Knowledge }\end{array}$ & $\begin{array}{l}\text { Forces Mandate With Required } \\
\text { Entry, Free Text Hard Retrieve Use } \\
\text { Free Text Difficult Forces Manual } \\
\text { Billing, Time Required Review Text } \\
\text { Text Justifies Dr Effort, Not } \\
\text { Physician Centric }\end{array}$ & 10 \\
\hline Collaboration & 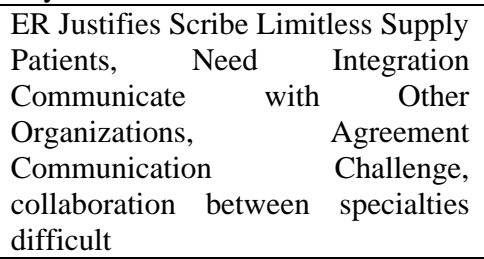 & 9 \\
\hline $\begin{array}{l}\text { Restriction to } \\
\text { Activation of } \\
\text { Discretionary } \\
\text { Knowledge }\end{array}$ & $\begin{array}{l}\text { Technology Requirements Not } \\
\text { Natural Work, No Logical Flow, } \\
\text { Developed by IT Machine Centric, } \\
\text { Machine Over Man, Not Intuitive To } \\
\text { Support Work, Technology Distracts } \\
\text { Natural Flow Thought, Irrelevant } \\
\text { Everything Slows Down Dr, } \\
\text { Reliability Issue, Computer Before } \\
\text { Patient Interaction, Forced Question } \\
\text { Sequence Wrong Order Thought }\end{array}$ & 26 \\
\hline $\begin{array}{l}\text { Demand for } \\
\text { Action }\end{array}$ & $\begin{array}{l}\text { Need Search Retrieve, Would Like } \\
\text { Voice Recognition, Wants Auto Tag } \\
\text { Of Abnormal Results, Wants } \\
\text { Automatic Analysis, Clinical Alerts } \\
\text { There Now, Want Therapist Session } \\
\text { Voice Capture, Need Training } \\
\text { Understand And Utilize, Back } \\
\text { Basics Want Reliability Integration }\end{array}$ & 27 \\
\hline Diagnosis & $\begin{array}{l}\text { IT Data Quality Mismatch Quality } \\
\text { Patient Care, Technology Forces } \\
\text { Data Driven Approach, System } \\
\text { Detracts From Patient Assessment }\end{array}$ & 7 \\
\hline $\begin{array}{lr}\text { Search } & \text { and } \\
\text { Evaluation } & \text { of } \\
\text { Treatment } & \\
\text { options } & \end{array}$ & $\begin{array}{l}\text { Clinician Required Search 5Minutes } \\
\text { Find Orderable, Physician } \\
\text { Reminders Force Complete Data } \\
\text { When Not Necessary for Patient Or } \\
\text { Care }\end{array}$ & 5 \\
\hline $\begin{array}{l}\text { Implementation } \\
\text { of Treatment }\end{array}$ & $\begin{array}{lcc}\text { Inconsistent } & \text { Data Entry } & \text { Time } \\
\text { Results } & \text { Perception } & \text { Poor } \\
\text { Communication, Pharmacy Biggest } \\
\text { Communication } \\
\text { Challenge, EMR Does not Integrate } \\
\text { Across Facilities ,Patient Picture Not } \\
\text { Complete Due Lack Integration }\end{array}$ & 10 \\
\hline \multirow[t]{2}{*}{$\begin{array}{l}\text { Assessment and } \\
\text { Follow-up }\end{array}$} & $\begin{array}{l}\text { System Work Flow mismatch, No } \\
\text { access to patient information at } \\
\text { follow-up, IT Systems Compliant? }\end{array}$ & 6 \\
\hline & Total & 100 \\
\hline
\end{tabular}

The majority of the labels related to demands for action (27), particularly for better functionality in a health information system. The second largest number of labels pertained to restrictions to the activation of discretionary knowledge (26). This suggests that while accountable knowledge (10) is important for the provision of 
healthcare, the need to activate discretionary knowledge is also very important. We did not find any labels pertaining to the activation of autonomous knowledge.

As can be seen from the above label frequencies, collaboration is limited to 9 labels, diagnosis was found only 7 times, search and evaluation of treatment options only 5 times, implementation of treatment 10 times and assessment and follow-up 6 times. It appears that the majority of activities that require patient centered care takes place outside of the system. It appears that the design of the system would have to include additional functionalities to activate discretionary and autonomous knowledge, if it is to support patient centered care.

\section{Contributions to Theory and Practice}

This paper makes a contribution to the theory of knowledge activation by offering concepts that relate to the use of HIT in the provision of patient centered care. We use grounded theory analysis to discover conceptual categories of knowledge activation through the processes of diagnosis, search and evaluation of treatment options, implementation of treatment, and the assessment and follow-up with the patient. Through a grounded theory analysis of focus group sessions, we discover the knowledge activation processes that take place and those that are hindered. We uncover the dichotomy between patient centered care and evidence based care as it relates to the activation of knowledge.

While the healthcare provision process is very collaborative, the results of this research have shown that the EHRs tend to limit the activation of discretionary and autonomous knowledge identities. This is because the EHRs limits the extent to which purposeful joint action can take place. Discretionary and autonomous knowledge identities need to be activated for patient centered care. The EHRs support the activation of accountable knowledge. Accountable knowledge is used in evidence based care.

This study contributes to practice by identifying the knowledge activation processes that may help overcome the divide between patient and evidence based care and by identifying the struggle between evidence based care and patient centered care. The finding that EHRs may be a detriment to the activation of discretionary and autonomous knowledge which does not fit into any of the pre-defined fields of EHRs is a significant contribution to the field of HIT and warrants further research.

\section{Conclusions and Future Research}

This paper has investigated the physician - patient divide that is currently being widened due to a dichotomy between evidence based care and patient centered care. Following an analysis of physician interviews regarding their use of electronic health records, opportunities for bridging the physician patient divide, we found that the EHR is actually a detriment to the activation of knowledge, in particular, discretionary and autonomous knowledge which does not fit into any of the pre-defined fields of the system. These are the most important processes that need to be supported to bridge the HIT divide.

The limitations of this research are many. Further research will have to be conducted work is need to refine and clarify the gap between patient centered and evidence based care. The analysis can be strengthened with additional data to show how this research contributes to existing knowledge management theories. This would enable discovery of key drivers currently in the healthcare domain and develop a more robust theoretical lens to guide this line of inquiry.

\section{References}

[1] Agarwal, R., Gao, G., and DesRoches, C. (2010), "Research Commentary: The Digital Transformation of Healthcare: Current Status and the Road Ahead”. Information Systems Research 21(4), pp. 796-809

[2] Anderson, J. \& Aydin, C. (1997). Evaluating the impact of health care information systems. International Journal Technical Assessment in Healthcare, 13(2), 380-393.

[3] Anderson, J. (1997). Clearing the way for physicians' use of clinical information systems. Communications of the ACM, 40(8), 83-90.

[4] Ash, J. S. (1997). "Factors affecting the diffusion of the computer-based patient record.” Journal of the American Medical Informatics Association Supplement, AMIA Proceedings, 682-686.

[5] Barry, M. J., \& Edgman-Levitan, S. (2012). Shared decision making - the pinnacle of patient-centered care. New England Journal of Medicine, 366(9), 780-781.

[6] Bensing, J. (2000). Bridging the gap.: The separate worlds of evidence-based medicine and patient-centered medicine. Patient education and counseling, 39(1), 17-25.

[7] Blumenthal, D. and M. Tavenner (2010), “The 'Meaningful Use' Regulation for Electronic Health Records” The New England Journal of Medicine. 363(6), pp501-504.

[8] Cleveringa, F. G., Gorter, K. J., van den Donk, M., van Gijsel, J., \& Rutten, G. E. (2013). Computerized decision support systems in primary care for type 2 diabetes patients only improve patients' outcomes when combined with feedback on performance and case management: a systematic review. Diabetes technology \& therapeutics, 15(2), 180-192. 
[9] Cliff, B. (2012). Using Technology to Enhance PatientCentered Care. Journal Of Healthcare Management, 57(5), 301-303.

[10] Clifton, J. (2012). Healthcare is killing us. http://businessjournal.gallup.com/content/151862/Hea lthcare-

killing.aspx?utm_source=add+this\&utm_medium= addthis.com\&utm_campaign=sharing

[11] DesRoches C.M., Campbell E.G., Vogeli C., Zheng J, Rao S.R., Shields A.E., Donelan K., Rosenbaum S., Bristol S.J. and A. K. Jha, (2010) "Electronic Health Records' Limited Successes Suggest More Targeted Uses” Health Affairs. vol. 29 no. 4 639-646.

[12] Fontaine, P. Ross, S.E., Zink, T. Schilling, L.M. 2010. "Systematic Review of Health Information Exchange in Primary Care Practices", Journal of the American Board of Family Medicine (23), pp. 655- 670.

[13] Gabriel, S. E., and S. T. Normand, August 30, 2012, "Getting the Methods Right-The Foundation of Patient -Centered Outcomes Research.” NEJJM. 367:787-790.

[14] Glaser, B. S., \& Strauss, A. (1967). The discovery of grounded theory. Strategies for qualitative research. London: Weidenfeld and Nicolson.

[15] Hirsch, A. G., \& McAlearney, A. S. (2014). Measuring Diabetes Care Performance Using Electronic Health Record Data: The Impact of Diabetes Definitions on Performance Measure Outcomes. American Journal of Medical Quality, 29(4), 292-299

[16] Institute of Medicine, Committee on Health Care in America (IOM). (2001). Crossing the quality chasm: A new health system for the 21st Century. Washington, DC: National Academy Press.

[17] Kane, G. and G. Labianca. (2011). "IS Avoidance in Health-Care Groups: A Multilevel Investigation.” Information Systems Research 22(3), pp. 504-522.

[18] Makary, M. and M. Daniel., (2016), "Medical errorthe third leading cause of death in the US”. The British Medical Journal. 353 doi: http://dx.doi.org/10.1136/bmj.i2139.

[19] Miller, R. H., \& Sim, I. (2004). Physicians' use of electronic medical records: Barriers and solutions. Health Affairs, 23(2), 116-126.

[20] Murphy, J. (2011). Information Systems \& Technology. Patient as Center of the Health Care Universe: A Closer Look at Patient-Centered Care. Nursing Economic\$, 29(1), pp. 35-37.

[21] Noteboom, C. \& Qureshi, S., (2014) “Adaptations of electronic health records to activate physicians' knowledge: how can patient centered care be improved through technology?" Health and Technology. DOI 10.1007/s12553-013-0072-5

[22] Noteboom, C. and J. Hafner (2015) "Challenges of Change: Technological Ease or Technological Upset?” Twenty-first Americas Conference on Information Systems, Puerto Rico, 2015.

[23] Oates, J., Weston, W. W., \& Jordan, J. (2000). The impact of patient-centered care on outcomes. Family Practise, 49, 796-804.

[24] Pardue, H., Campbell, A., Campbell, M., and W. Pamela (2014) "Mission Impossible? Putting the Patient Back in Patient Care," Communications of the
Association for Information Systems: Vol. 34, Article 19. Available at: http://aisel.aisnet.org/cais/vol34/iss1/19

[25] Qureshi, S. and P. Keen. “Activating Knowledge through Electronic Collaboration: Vanquishing the Knowledge Paradox". IEEE Transactions in Professional Communication. Vol 48, Issue 1. Pp: 40- 54, 2005.

[26] Qureshi, S.; Noteboom, C.; Schumaker, A.M., (2015)."Mobile Access for Patient Centered Care: The Challenges of Activating Knowledge through Health Information Technology," System Sciences (HICSS), 2015 48th Hawaii International Conference on Information Sciences, pp.3227,3236, 5-8 Jan. 2015

[27] Randall D. Cebul, M.D., Thomas E. Love, Ph.D., Anil K. Jain, M.D., and Christopher J. Hebert, M.D. (2011) "Electronic Health Records and Quality of Diabetes Care" New England Journal Med 2011; 365:825-833September 1, 2011DOI: 10.1056/NEJMsa1102519

[28] Robinson, J. H., Callister, L.C., Berry, J.A.,and K.A. Dearing, (2008)."Patient-centered care and adherence: Definitions and applications to improve outcomes” Journal of the American Academy of Nurse Practitioners. Volume 20, Issue 12, pp. 600-607, December 2008

[29] Sackett DL, Rosenberg WMC, Gray JA, Haynes RB, Richardson A, WS. (1996)."Evidence-based medicine: what it is, and what it isn't.” British Medical Journal. pp312:71-2.

[30] Sacristán, J. A. (2013). Patient-centered medicine and patient-oriented research: improving health outcomes for individual patients. BMC Medical Informatics \& Decision Making, 13(1), 1-8. doi:10.1186/1472-6947-13-6

[31] Stewart, M. and J. Brown, A. Donner, I. McWhinney , J. (2000). "The impact of patient-centered care on outcomes." Journal of Family Practice, 49(9).

[32] Urquhart, C. (2013). Grounded theory for qualitative research: A practical guide. Sage. 